\title{
FOXQ1 promotes gastric cancer metastasis through upregulation of Snail
}

\author{
JING ZHANG $^{1 *}$, YIMIN LIU $^{2 *}$, JIA ZHANG $^{1 *}$, XIAOHAI CUI ${ }^{1}$, GANG LI $^{1}$, \\ JIANSHENG WANG $^{1}$, HONG REN ${ }^{1}$ and YUNFENG ZHANG ${ }^{1}$ \\ ${ }^{1}$ The Second Department of Thoracic Surgery, First Affiliated Hospital of Xi'an Jiaotong University, \\ Xi'an, Shaanxi 710061; ${ }^{2}$ Baoji Renmin Hospital, Baoji, Shaanxi 721000, P.R. China
}

Received December 25, 2015; Accepted February 15, 2016

DOI: $10.3892 /$ or.2016.4736

\begin{abstract}
Gastric cancer (GC) is one of the most common cancers, and the second most common cause of cancer deaths worldwide. Forkhead box Q1 (FOXQ1) is a member of the forkhead transcription factor family and its upregulation is closely correlated with tumor progression and prognosis of multiple cancer types, including GC. FOXQ1 has been shown to regulate EMT and function in human cancers. However, the role of FOXQ1 in regulating EMT in GC and the exactly mechanism has not been clarified. The purpose of this study was to investigate the effects of FOXQ1 on EMT in human GC. FOXQ1 protein was detected by immunohistochemistry in human GC specimens and their clinical significance evaluated. We examined the cell biology and molecular biology changes after overexpression and knockdown of FOXQ1 in gastric cancer cells in vitro. To further understand the underlying mechanisms of EMT promoted by FOXQ1, we examined the changes of target genes of FOXQ1 after overexpression and knockdown of FOXQ1 in gastric cancer cells. In the present study, we demonstrate that FOXQ1 is overexpressed in GC tissues and its expression level is closely correlated with histologic differentiation, pTNM stage, and lymphatic metastasis of GC. Kaplan-Meier survival analysis showed that a high expression level of FOXQ1 resulted in a significantly poor prognosis of GC patients. FOXQ1 modulated GC cell invasion in vitro, and induced E-cadherin repression. FOXQ1 also upregulated the expression of vimentin in vitro. The Snail signaling pathway was likely involved in the induc-
\end{abstract}

Correspondence to: ProfessorHong RenorProfessor YunfengZhang, The Second Department of Thoracic Surgery, First Affiliated Hospital of Xi'an Jiaotong University, 277 West Yanta Road, Xi'an, Shaanxi 710061, P.R. China

E-mail: medicinedr.ren@gmail.com

E-mail: zhangjiaxjtu@xjtu.edu.cn

*Contributed equally

Key words: gastric cancer, FOXQ1, epithelial-mesenchymal transition, E-cadherin, vimentin, invasion tion of EMT by FOXQ1 in GC. Our results demonstrate that FOXQ1 is a prognostic marker for patients with GC, FOXQ1 overexpression is involved in acquisition of the mesenchymal phenotype of gastric cancer cells, and that subsequent Snail expression is essential for induction of EMT. The results suggest that FOXQ1 is a potential therapeutic target for the development of therapies for GC.

\section{Introduction}

Gastric cancer (GC) is one of the most common cancers, and the second most common cause of cancer deaths worldwide (1). Despite improvements in diagnostic and treatment strategies, the prognosis for advanced stage GC patients is still very poor (2). This is mainly because of the high incidence of metastasis and recurrence in GC patient (3). Therefore, a better understanding of the molecular mechanisms involved in the progression of GC is important for the development of novel therapeutic strategies for the treatment of patients with GC.

In 1968, Elizabeth Hey first defined the concept of epithelial-mesenchymal transition (EMT). It is an essential embryonic process during which epithelial cells loose contact with their neighbors and gain mesenchymal properties, this could enable them to break through the basement membrane which separates different tissues from the embryo (4). In the last few years, an increasing number of studies reported that aberrant reactivation of EMT could promote tumor cell migration and invasion by disruption of apical-basal polarity and loss of E-cadherin expression $(5,6)$. Increasing evidence have revealed that many growth factors, cytokines and cellular signaling pathways play important roles in initiation and execution of EMT, including TGF- $\beta$, FoxM1, HGF, EGF, NFאB, Notch, Snail, ZEB1, ZEB2, Twist1, KLF4, KLF8, Sox9 and Wnt (5,7-10). Thus, it is essential to clarify the possible signaling pathways in EMT of GC.

Forkhead box Q1 (FOXQ1, also known as HFH1) is a new member of the forkhead (FOX) transcription factor family. It contains the core DNA binding domain, whereas the flanking wings of FOXQ1 contribute to its sequence specificity (11). FOXQ1 has been shown to repress the promoter activity of smooth muscle-specific genes such as SM22 $\alpha$ and telokin in A10 cells (12). A previous study reported that FOXQ1 plays critical roles in hair follicle morphogenesis and gastric epithe- 
lial differentiation (13). Increasing evidence indicates FOXQ1 overexpression in different human cancers, including colorectal cancer, esophageal cancer, hepatocellular carcinoma, breast cancer, and non-small cell lung cancer, and its upregulation enhances tumor growth and invasion (14-23). FOXQ1 has been shown to regulate EMT and function in breast cancer, nonsmall cell lung cancer, colorectal cancer and hepatocellular carcinoma $(15,18,19,21,23)$. Overexpression of FOXQ1 has been reported to be strongly correlated with poor prognosis in hepatocellular carcinoma, non-small cell lung cancer, and GC $(18,20,24)$. To date, scarce data exist regarding the cellular and biological functions of FOXQ1 in GC except a recent study reporting the upregulation of FOXQ1 involving miR-1271 (25).

In the present study, we examined both the mRNA and protein expression levels of FOXQ1 in GC tissue samples and cell lines and further analyzed the clinical significance of FOXQ1 expression in a cohort of GC patients. Moreover, our present data suggest that FOXQ1 promotes Snail expression to induce EMT-like metastatic progression in GC.

\section{Materials and methods}

Tissues and cells. We collected tissues specimens of 60 gastric cancer patients from consecutive surgical cases in Department of Surgical Oncology, The First Affiliated Hospital, Xi'an Jiaotong University between 2004 and 2009. The patients included 37 male and 23 female patients (ranging from 45 to 68 years of age). All of the patients were assessed according to the system for staging primary tumor/regional lymph nodes/distant metastasis (TNM) described in the AJCC Cancer Staging Manual. None of these 60 patients received neoadjuvant or adjuvant chemotherapy before the operation. Paraffin-embedded tumor specimens were carefully collected immediately after the surgery. The clinicopathological characteristics of the patients are summarized in Table I. This study was approved by the Protection of Human Subjects Committee of First Affiliated Hospital, Xi'an Jiaotong University and complies with the Helsinki declaration. The BGC-823, MKN-45, SGC-7901, AGS, MGC-803, and GES1 cell lines used were cultured in RPMI-1640 medium supplemented with $10 \%$ fetal bovine serum (HyClone, Logan, UT, USA).

Immunohistochemical staining. The tissues specimens were fixed in neutral buffered formalin and embedded in paraffin wax. The 4-mm sections were cut and mounted on charged glass slides. Antigen retrieval was performed using citrate buffer at $\mathrm{pH}$ 6.0. Immunohistochemical staining was performed with rabbit anti-human FOXQ1 (bs-16175R, Beijing Bioss Biotechnology, Beijing, China). The streptavidinperoxidase technique (SP-9001 Golden Bridge Int., Beijing, China) was used. An irrelevant rabbit antiserum served as a negative control. The sections were stained with $0.02 \%$ diaminobenzidine (DAB) solution followed by counterstaining with hematoxylin.

Evaluation of immunohistochemical analysis. The evaluation of FOXQ1 expression was performed independently by two experienced pathologists who were blinded to the clinical data with consensus. Sections were observed under a light microscope (Carl Zeiss Axio Scope A1 microscope) at high
Table I. Correlation between FOXQ1 expression with clinicopathological variables of gastric cancer patients.

\begin{tabular}{lrrrr}
\hline & & \multicolumn{2}{c}{ FOXQ1 } & \\
\cline { 3 - 3 } Variables & Total & Low & High & P-value \\
\hline Age & & & & 0.794 \\
$<60$ & 35 & 14 & 21 & \\
$\geq 60$ & 25 & 9 & 16 & \\
Gender & & & & 0.787 \\
$\quad$ Male & 37 & 15 & 22 & \\
Female & 23 & 8 & 15 & \\
Smoking & & & & 1.000 \\
$\quad$ Yes & 46 & 18 & 28 & \\
No & 14 & 5 & 9 & \\
Alcohol consumption & & & & 0.544 \\
$\quad$ Yes & 45 & 16 & 29 & \\
$\quad$ No & 15 & 7 & 8 & \\
Histologic & & & & \\
differentiation & & & & $0.000^{\mathrm{a}}$ \\
$\quad$ Well & 10 & 7 & 3 & \\
Moderately & 37 & 16 & 21 & \\
Poorly & 13 & 0 & 13 & \\
TNM stages & & & & $0.014^{\mathrm{a}}$ \\
I-II & 24 & 14 & 10 & \\
III-IV & 36 & 9 & 27 & \\
Lymphatic metastasis & & & & $0.001^{\mathrm{a}}$ \\
$\quad$ No & 23 & 15 & 8 & \\
$\quad$ Yes & 37 & 8 & 29 & \\
\hline & & & & \\
\hline
\end{tabular}

${ }^{a} \mathrm{P}<0.05$

magnification (x400). The staining results of FOXQ1 were scored semi-quantitatively by calculating the immunostaining intensity and the percentage of positive malignant cells. The percentage of positive malignant cells was determined in at least 5 areas under $\mathrm{x} 400$ magnifications and averaged. The mean percentage was scored as: $0(0-5 \%) ; 1(6-25 \%)$; 2 (26-50\%); $3(51-75 \%)$, and 4 (76-100\%). The staining intensities were scored as follows: no coloring, 0 point; slightly yellow, 1 point; brownish-yellow, 2 points; and tan, 3 points. Finally, the staining score was obtained by calculating the product of the staining intensity and the positive cell percentage, where $\leq 5$ was defined as low expression and $\geq 6$ as high expression.

Plasmids, antibodies, and reagents. The FOXQ1 shRNA lentiviral particle containing FOXQ1 shRNA sequences was purchased from Santa Cruz Biotechnology, Inc. (Santa Cruz, CA, USA), MKN-45 cells were infected with shFOXQ1 lentiviral particles and a negative control for $48 \mathrm{~h}$ and followed by $2 \mathrm{mg} / \mathrm{ml}$ puromycin selection. Full-length FOXQ1 cDNA was cloned into pCMV6/AC/GFP vector (OriGene, Rockville, MD, USA). Cell lines were separately transfected with plasmids and 
A

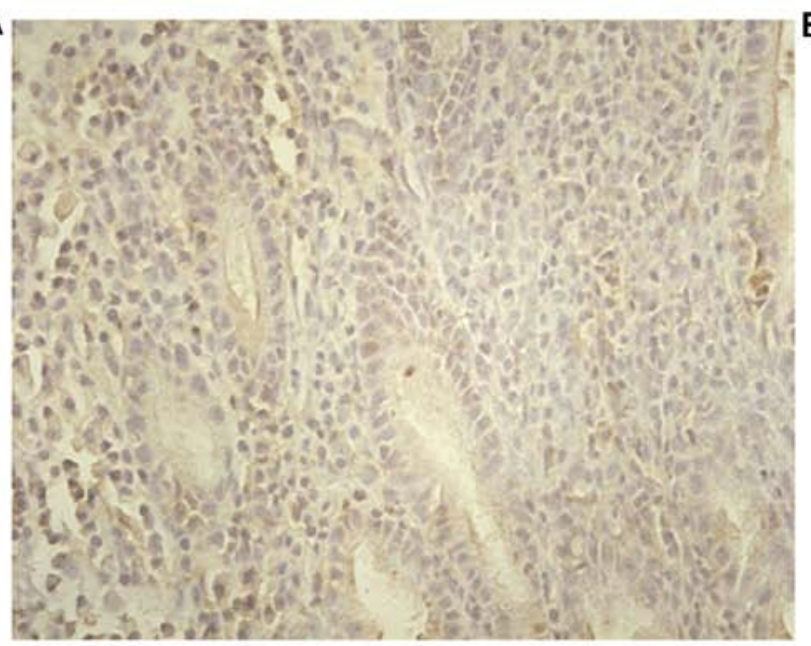

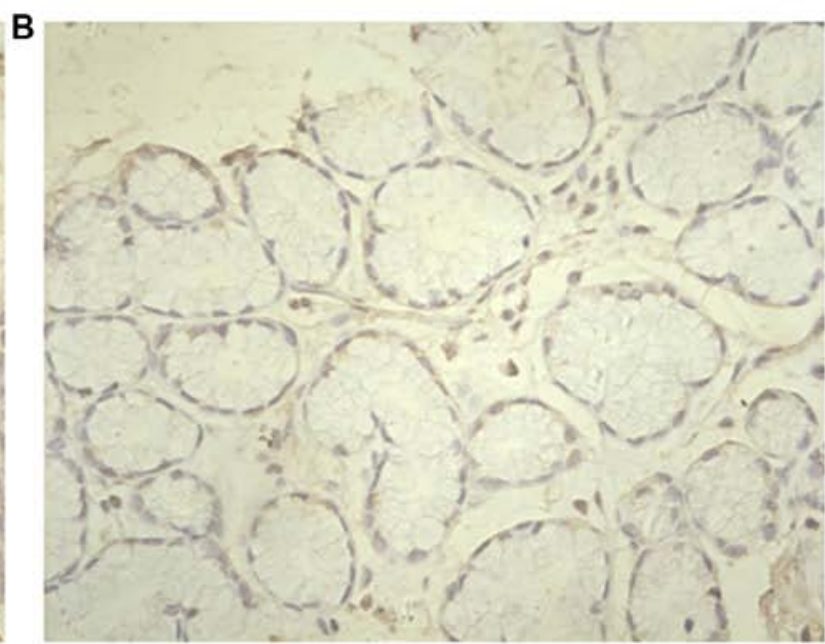

Figure 1. FOXQ1 is overexpressed in gastric cancer cells. FOXQ1 was stained in gastric cancer samples (A), adjacent non-tumorous tissues (B). Images were acquired at $\mathrm{x} 200$. Representative images are shown.

selected by GFP sorting. Cells were then grown in complete medium containing $200 \mu \mathrm{g} / \mathrm{ml} \mathrm{G} 418$ (Roche Diagnostics, Mannheim, Germany). qRT-PCR and western blotting was used to confirm the presence of the plasmids.

Quantitative RT-PCR ( $q R T-P C R)$. Total RNA was isolated using TRIzol reagent (Invitrogen, Carlsbad, CA, USA) and reverse transcribed into cDNA using the Reverse Transcription kit from Takara. After adjusting the cDNA concentration in all groups, qRT-PCR was performed using the CFX96 Real-time PCR Detection System (Bio-Rad, Hercules, CA, USA) with SYBR Green. The PCR conditions were as follows: pre-denaturation at $95^{\circ} \mathrm{C}$ for $30 \mathrm{sec}$; 35 cycles of denaturation $\left(95^{\circ} \mathrm{C}\right.$ for $\left.5 \mathrm{sec}\right)$, annealing $\left(55-60^{\circ} \mathrm{C}\right.$ for $30 \mathrm{sec})$ and extension $\left(72^{\circ} \mathrm{C}\right.$ for $\left.1 \mathrm{~min}\right)$; final extension at $72^{\circ} \mathrm{C}$ for $10 \mathrm{~min}$. The relative level of gene expression was calculated using the $\Delta \Delta \mathrm{Ct}$ method with normalization to GAPDH. All experiments were performed in triplicate. The primers used are listed in Table II.

Western blot analysis. Protein expression levels were analyzed by western blotting standard protocols. Briefly, $20 \mu \mathrm{l}$ of total protein extracts was resolved by denaturing sodium dodecyl sulfate-polyacrylamide gel electrophoresis and transferred to polyvinylidene difluoride membranes. The membranes were blocked with $5 \%$ non-fat milk and then incubated with primary antibodies against FOXQ1 (ab51340; Abcam, Cambridge, MA, USA), E-cadherin (AF0131; Affinity, USA), vimentin (5741; Cell Signaling Technology, Inc., Beverly, MA, USA) and $\beta$-actin (T0022; Affinity). The blots were washed and probed with the respective secondary peroxidase-conjugated antibodies. Signals were detected using the chemiluminescence solvent (Thermo Scientific, Rockford, IL, USA).

Cell invasion assay. Cell invasion was measured using Transwell chambers (Millipore, Billerica, USA) coated with Matrigel. Cells from different groups were suspended in serum free RPMI-1640 and were added into the upper compartment of the chamber; the bottom chamber was filled with RPMI-1640 containing 10\% FBS as a chemoattractant. After

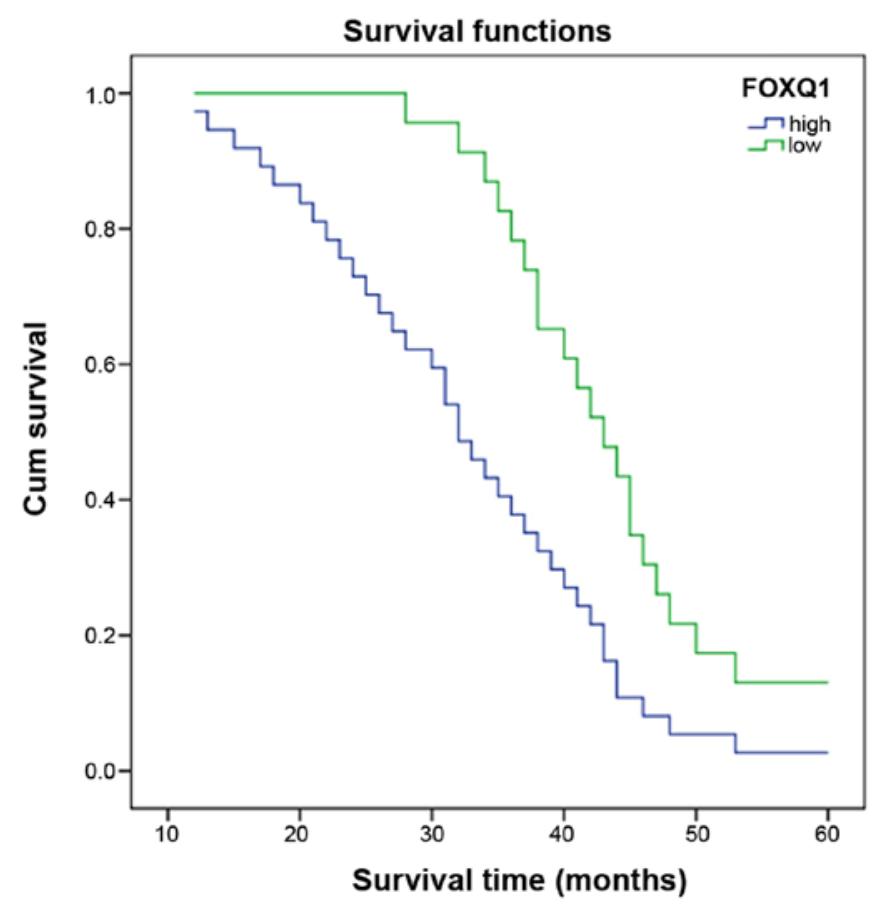

Figure 2. Overall survival (OS) differences between patients with high and low levels of FOXQ1 protein expression.

incubation in a $5 \% \mathrm{CO}_{2}$ humidified chamber at $37^{\circ} \mathrm{C}$ for $24 \mathrm{~h}$, the cells were removed from the upper surface of the filter with a cotton swab. The invaded cells were then fixed and stained using $0.1 \%$ crystal violet. The cells were quantified from five different fields under a light microscope. The experiment was repeated in triplicate.

Statistical analysis. Statistical analysis was done using the SPSS software package (version 16.0, SPSS Institute) or Prism (GraphPad Software, Inc., La Jolla, CA, USA). The association between staining index and other categorical factors potentially predictive of prognosis was analyzed using the Chi-square test and Fisher's exact test. Overall survival (OS) was defined as the time from the date of surgery to the date 
Table II. Primer sequences for qRT-PCR amplification of different genes.

\begin{tabular}{ll}
\hline Gene & \multicolumn{1}{c}{ Primer } \\
\hline GAPDH & F: 5'-CTTTGGTATCGTGGAAGGACTC-3' \\
& R: 5'-GTAGAGGCAGGGATGATGTTCT-3' \\
FOXQ1 & F: 5'-TGATTTCTTGCTATTGACCGATGC-3' \\
& R: 5'-GCCCAAGGAGACCACAGTTAGAG-3' \\
E-cadherin & F: 5'-TGGCTTCCCTCTTTCATCTCC-3' \\
& R: 5'-TCATAGTTCCGCTCTGTCTTTGG-3' \\
Vimentin & F: 5'-TCAATGTTAAGATGGCCCTTG-3' \\
& R: 5'-TGAGTGGGTATCAACCAGAGG-3' \\
Snail & F: 5'-GAC CAC TAT GCC GCG CTC TT-3' \\
& R: 5'-TCG CTG TAG TTA GGC TTC CGA TT-3'
\end{tabular}

F, forward; R, reverse.

of last follow-up or death from any cause. Survival curve and median survival were estimated by the Kaplan-Meier method. Their differences were verified by log-rank test. Differences between groups were assessed using an unpaired, two-tailed Student's t-test; $\mathrm{P}<0.05$ was considered significant.

\section{Results}

Expression of FOXQ1 in GC tissues and its significance. FOXQ1 protein level was examined in 60 gastric cancer tissues and their corresponding normal gastric epithelium tissues using immunohistochemistry. The clinicopathological characteristics of the gastric cancer patients are described in Table I. Respective photomicrographs of immunohistochemical staining of FOXQ1 are shown in Fig. 1. Increased FOXQ1 expression was detected in 37 of the 60 tumor tissue samples $(61.7 \%)$ and in $14(23.9 \%)$ of the 60 adjacent matched tumor tissues $\mathrm{P}<0.05$. High-expression of FOXQ1 was closely related to the histological differentiation, pTNM stage, and lymphatic metastasis, but not to the age, gender, smoking, or alcohol consumption of the patients.

To investigate the prognostic significance of FOXQ1 in GC, the correlation between FOXQ1 expression and overall survival time was assessed by Kaplan-Meier analysis along with log-rank test. Kaplan-Meier analysis showed that patients with higher FOXQ1 expression had significantly poorer prognosis than those with lower FOXQ1 expression (mean overall survival 32.0 vs. 43.0 months, $\mathrm{P}=0.002$, log-rank test; Fig. 2).

Expression of FOXQ1 in gastric epithelial cell lines of different origin. To determine if expression of FOXQ1 is increased in gastric cancer cell lines compared with human immortalized gastric epithelial cell line, we investigated the protein and mRNA level of FOXQ1 in five human gastric cancer cell lines (BGC-823, MKN-45, SGC-7901, AGS, MGC-803) and one human immortalized gastric epithelial cell line (GES-1). Western blot and qRT-PCR results showed higher expression of FOXQ1 in MKN-45 and AGS cell lines than BGC-823,

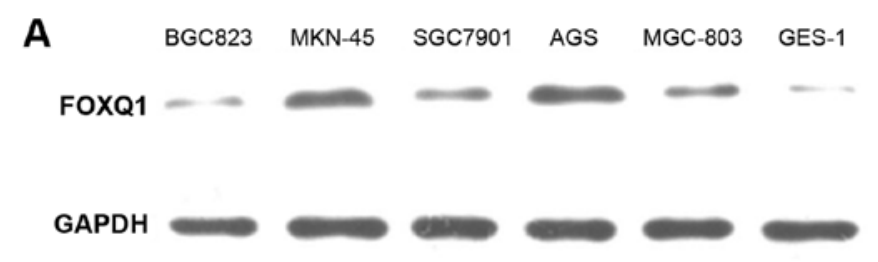

B

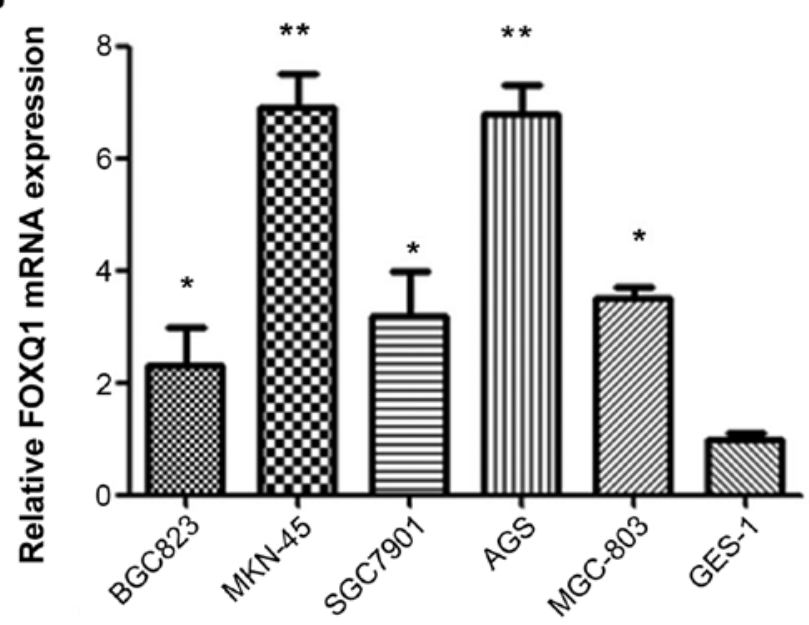

Figure 3. Expression of FOXQ1 in GC cell lines. Expression levels of FOXQ1 protein (A) and mRNA (B) in GC cell lines. ${ }^{*} \mathrm{P}<0.05,{ }^{* *} \mathrm{P}<0.01$.

SGC-7901, and MGC-803 cell lines compared to GES-1 cell line (Fig. 3). MKN-45 and BGC-823, with the highest and the lowest expression levels of FOXQ1, were chose for further investigation.

Overexpression of FOXQ1 in BGC-823 cell line results in $E M T$ and increased invasiveness. Overexpressing FOXQ1 expressed higher levels of mesenchymal marker, vimentin and lower levels of epithelial marker E-cadherin compared with their vector control at both protein and mRNA levels (Fig. 4A and B). To investigate whether the FOXQ1-induced EMT-like phenotype could be translated into enhanced metastatic ability of the BGC-823 cells, the invasion of BGC-823-FOXQ1 cells was tested. FOXQ1-overexpressing BGC-823 cells displayed increased motility through the Matrigel in Transwell invasion assays, compared with the vector control cells (Fig. 4C and D).

Knockdown of FOXQ1 in MKN-45 cell line inhibited EMT and decreased aggressiveness of the invasive gastric cancer cells. FOXQ1 was knocked down by shRNA in the high metastatic potential cell line MKN-45, which expresses high levels of FOXQ1. Knockdown of FOXQ1 in the cell line resulted in a significant decrease at both protein and mRNA level. Protein and mRNA expression of the mesenchymal marker vimentin was also decreased. The level of epithelial marker E-cadherin was increased (Fig. 5A and B). Furthermore, knockdown of FOXQ1 in MKN-45 cells resulted in decreased ability of the cells to invade through the Matrigel in the invasion assay (Fig. 5C and D).

Overexpression of FOXQ1 promotes Snail expression, whereas it is inhibited by FOXQ1 knockdown. To elucidate 
A

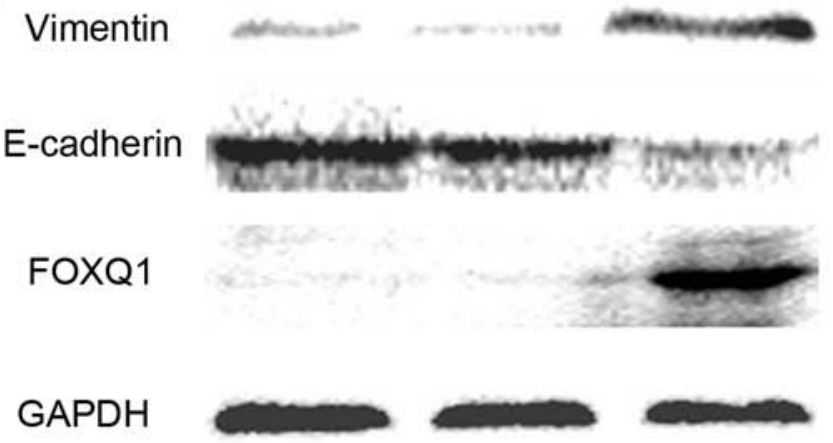

B
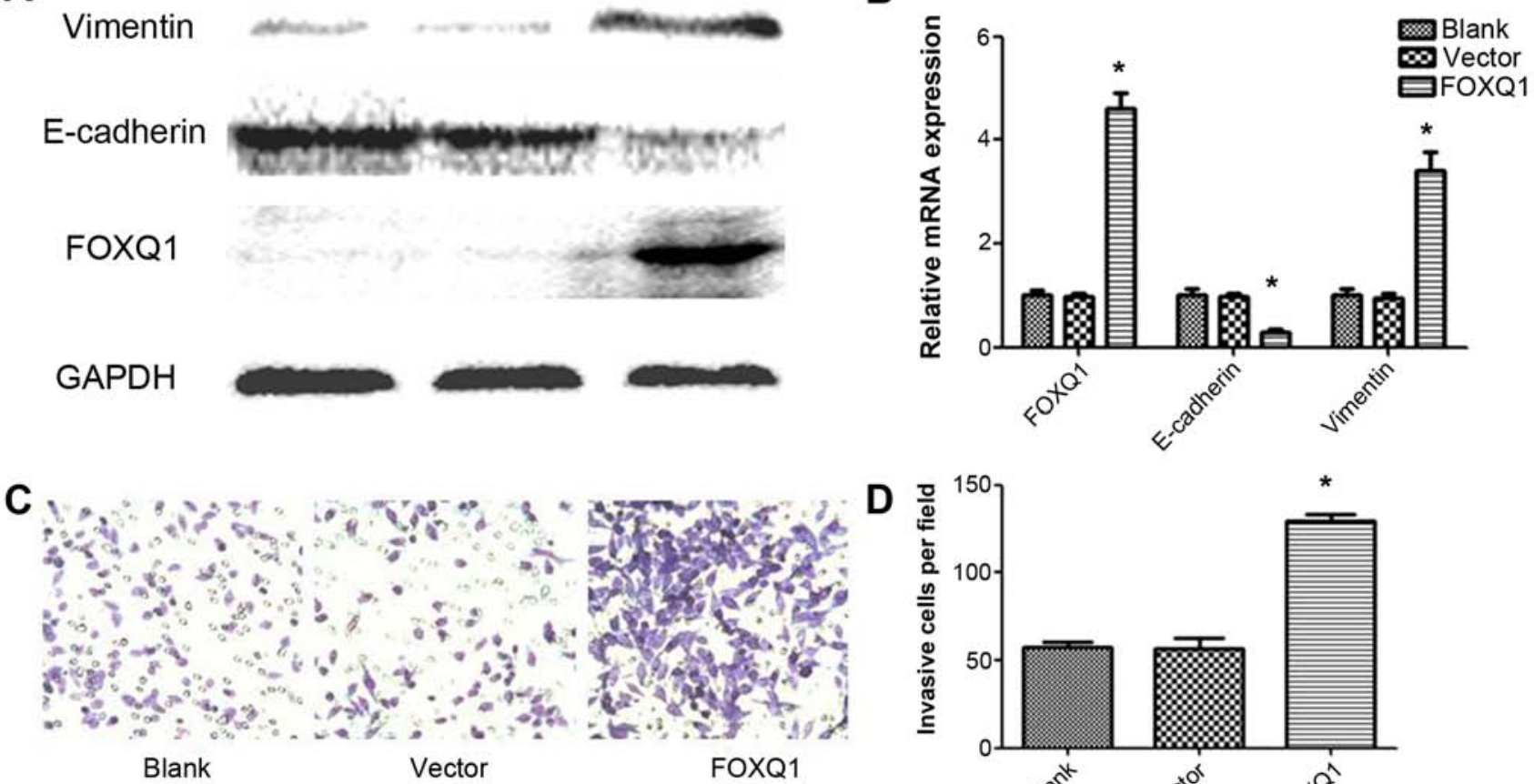

Figure 4. Overexpression of FOXQ1 in BGC-823 cell line resulted in EMT and increased invasiveness. To determine whether FOXQ1 promotes the EMT to increase cell invasion, the expression levels of FOXQ1, E-cadherin and vimentin were detected via western blotting and qRT-PCR. The results revealed that FOXQ1 could increase EMT, which decreased E-cadherin expression and increased vimentin expression ("P<0.05 vs. controls) (A and B). BGC-823 cells transfected with FOXQ1 expression plasmid or vector were subjected to Transwell invasion assays, the invasive cell numbers are the average count of five random microscopic fields detected using the Transwell invasion assay. Each bar represents the mean $\pm \mathrm{SD}$ of the counts $(\mathrm{C}$ and $\mathrm{D})$. ${ }^{*} \mathrm{P}<0.05$ compared with blank.

A

Vimentin

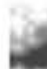

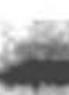
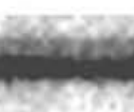

.

E-cadherin

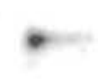

FOXQ1
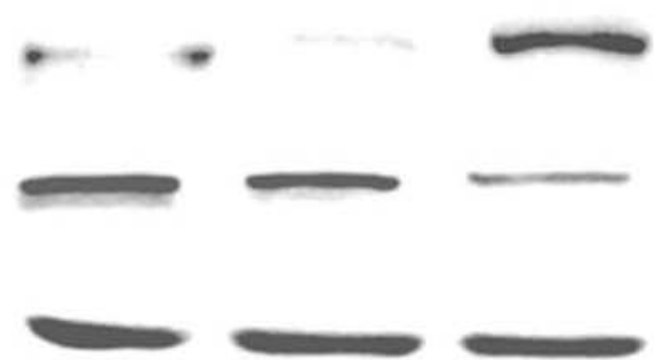

GAPDH

\section{C}

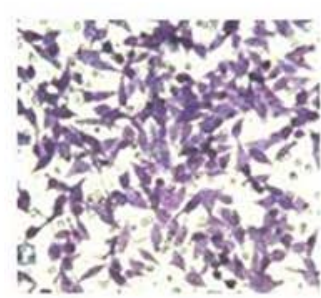

Blank
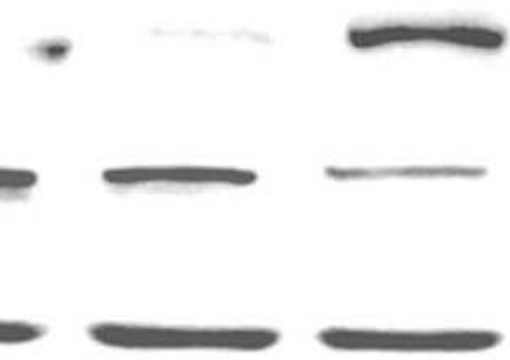

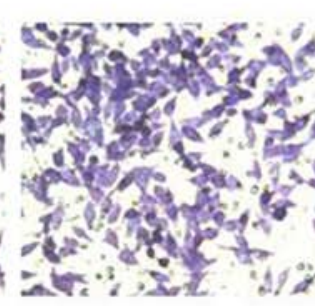

shNC

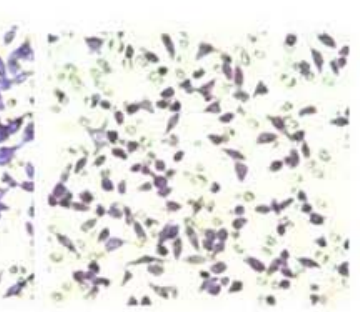

shFOXQ1
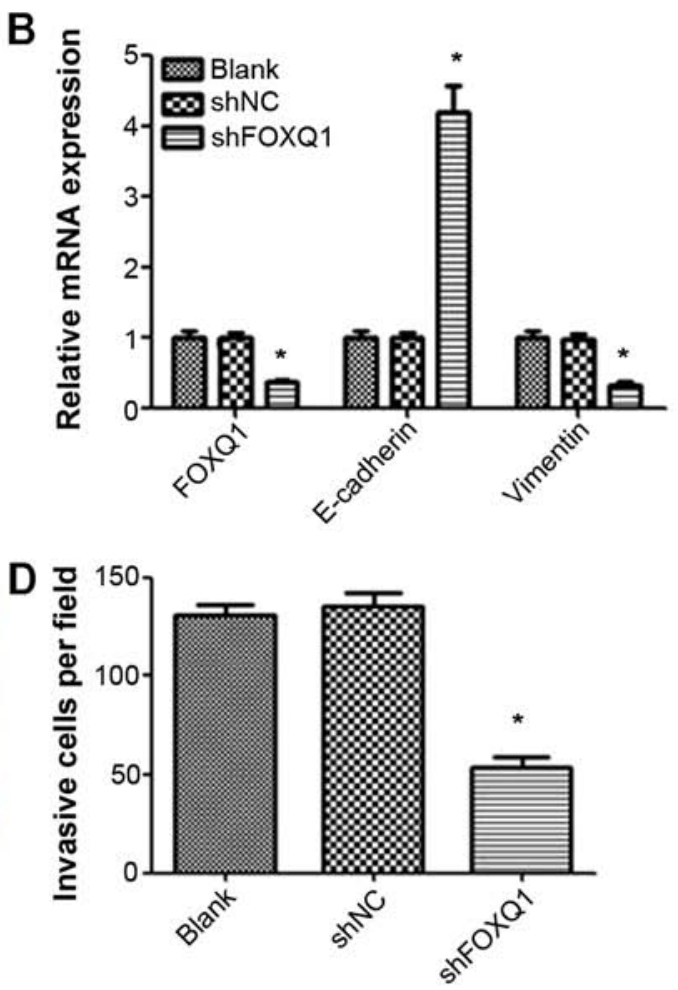

Figure 5. Knockdown of FOXQ1 in MKN-45 cell line inhibits EMT and decreases aggressiveness of the invasive gastric cancer cells. To determine whether knockdown of FOXQ1 inhibits EMT to decrease cell invasion, the expression levels of FOXQ1, E-cadherin and vimentin were detected via western blotting and qRT-PCR. The results revealed that knockdown of FOXQ1 could decrease EMT, which increased E-cadherin expression and decreased vimentin expression ( ${ }^{*} \mathrm{P}<0.05$ vs. controls) (A and B). MKN-45 cells transfected with shRNA-FOXQ1 or control shRNA were subjected to Transwell invasion assays, the invasive cell numbers are the average count of five random microscopic fields detected using the Transwell invasion assay. Each bar represents the mean \pm SD of the counts $(\mathrm{C}$ and $\mathrm{D}) .{ }^{*} \mathrm{P}<0.05$ compared with blank. 
A Snail

GAPDH

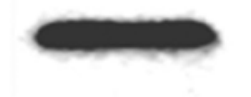

Blank Vector FOXQ1

C

\section{GAPDH}

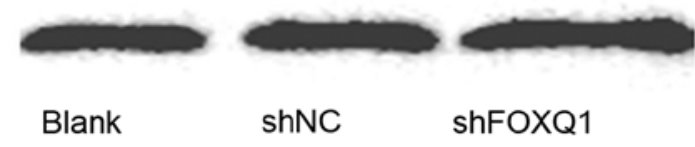

B

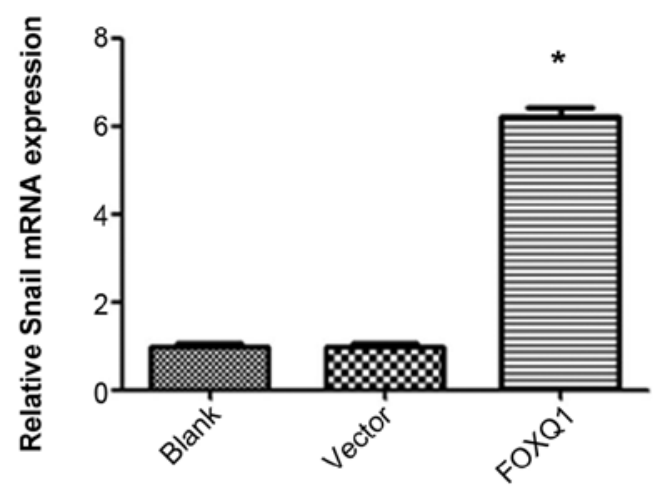

D

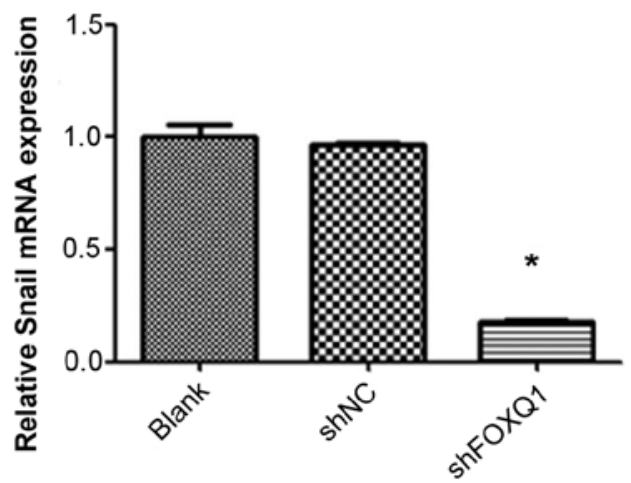

Figure 6. Overexpression of FOXQ1 promotes Snail expression, but it is inhibited by knockdown of FOXQ1. Overexpression of FOXQ1 increased the expression of Snail at both protein and mRNA level in BGC-823 cells (A and B). Knockdown of FOXQ1 decreased the expression of Snail at both protein and mRNA levels in MKN-45 cells (C andD).

the mechanism of FOXQ1 that leads to EMT and invasiveness, we hypothesized that FOXQ1 regulates the expression of Snail. Ectopic FOXQ1 expression greatly increased Snail at both the mRNA and the protein levels (Fig. 6A and B). Conversely, knockdown of FOXQ1 reduced Snail expression (Fig. 6C and D).

\section{Discussion}

To our knowledge, this is the first study to show that FOXQ1 induces epithelial-mesenchymal transition and promotes metastasis in gastric cancer. FOXQ1 overexpression in BGC-823 cells induced EMT and invasive in vitro. In contrast, in MKN-45 cell silencing of FOXQ1 reversed these events in otherwise aggressive and invasive GC cells. Furthermore, we demonstrated the mechanism by which FOXQ1 increases Snail expression. Indicating the role of FOXQ1 in regulating Snail expression and consequently EMT.

Accumulating evidence suggests that FOXQ1 is differentially expressed in human cancers (14-24). FoxQ1 has been shown to regulate EMT and function in human cancers $(15,18,19,21,23)$. Overexpression of FOXQ1 has been reported to be strongly correlated with poor prognosis in human cancer including GC $(18,20,24)$. A recent study indicated miR-1271 is upstream of FOXQ1 and could inhibit cell proliferation, invasion and EMT in GC by targeting FOXQ1 (25). However, the role of FOXQ1 in regulating EMT in $\mathrm{GC}$ and the exact mechanism has not been clarified.

In the present study, we observed FOXQ1 in $61.7 \%$ of tumor samples from GC patients and in $23.9 \%$ of adjacent matched tumor tissues. High-expression of FOXQ1 was closely related to the histologic differentiation $(\mathbf{P}=\mathbf{0 . 0 0 0}$ ?), pTNM stage $(\mathrm{P}=0.014)$, and lymphatic metastasis $(\mathrm{P}=0.001)$, but not to the patients' age, gender, smoking, and alcohol consumption. Consistent with previous studies, Kaplan-Meier analysis showed that patients with higher FOXQ1 expression had significantly poorer prognosis than those with lower FOXQ1 expression (mean overall survival 32.0 vs. 43.0 months, $\mathrm{P}=0.002$, log-rank test) (24).

Aberrant reactivation of EMT could promote tumor cell migration and invasion $(5,6,26)$. To reveal the exact role of FOXQ1 in regulating EMT in GC, we tested the effect of FOXQ1 on EMT makers and invasion by modulating the expression level of FOXQ1 using FOXQ1-shRNA and FOXQ1 plasmids. BGC-823 cells with ectopic expression of FOXQ1 displayed an EMT phenotype, including the associated stimulatory effect on invasion in vitro. While in MKN-45 cells silencing FOXQ1 displayed the reversal of the expression of epithelial marker E-cadherin and mesenchymal marker vimentin, including the associated inhibitory effect on invasion in vitro. The results suggested the novel function of FOXQ1 in GC metastasis through regulating EMT.

The roles of several growth factors, cytokines and cellular signaling pathways as EMT regulators have been extensively reported, including TGF- $\beta$, FoxM1, HGF, EGF, NFкB, Notch, Snail, ZEB1, ZEB2, Twist1, KLF4, KLF8, Sox9 and Wnt (5,7-10). As a critical EMT regulator, the expression of Snail is closely associated with cancer metastasis. Snail can bind to the E-box site in the promoter of E-cadherin and trigger the EMT of many types of cancer (27). In the present study, we showed that FOXQ1 overexpression enhanced the expression of Snail in BGC-823 cells, whereas FOXQ1 down- 
regulation had the opposite effect. These preliminary results reveal that the Snail signaling pathway is likely involved in FOXQ1-mediated EMT of human GC cells.

Overall, the present study shows that FOXQ1 is a prognostic marker for patients with GC, FOXQ1 overexpression is involved in acquisition of the mesenchymal phenotype of gastric cancer cells, and that subsequent Snail expression is essential for induction of EMT. The results suggest that FOXQ1 is a potential therapeutic target for the development of therapies for GC.

\section{Acknowledgements}

This work was supported by the Scientific and Technological Planning project of Shaanxi Province (no. 2014KW23-02) and the Fundamental Research Funds for the Central Universities.

\section{References}

1. Torre LA, Bray F, Siegel RL, Ferlay J, Lortet-Tieulent J and Jemal A: Global cancer statistics, 2012. CA Cancer J Clin 65: 87-108, 2015

2. Piazuelo MB and Correa P: Gastric cancer: Overview. Colomb Med (Cali) 44: 192-201, 2013.

3. Steeg PS: Metastasis suppressors alter the signal transduction of cancer cells. Nat Rev Cancer 3: 55-63, 2003.

4. Kalluri R and Weinberg RA: The basics of epithelial-mesenchymal transition. J Clin Invest 119: 1420-1428, 2009.

5. Thiery JP, Acloque H, Huang RY and Nieto MA: Epithelialmesenchymal transitions in development and disease. Cell 139: 871-890, 2009

6. Thiery JP and Sleeman JP: Complex networks orchestrate epithelial-mesenchymal transitions. Nat Rev Mol Cell Biol 7: 131-142, 2006

7. Grego-Bessa J, Díez J, Timmerman L and de la Pompa JL: Notch and epithelial-mesenchyme transition in development and tumor progression: Another turn of the screw. Cell Cycle 3: 718-721, 2004.

8. Kang Y and Massagué J: Epithelial-mesenchymal transitions: Twist in development and metastasis. Cell 118: 277-279, 2004.

9. Fuxe J, Vincent $\mathrm{T}$ and Garcia de Herreros A: Transcriptional crosstalk between TGF- $\beta$ and stem cell pathways in tumor cell invasion: Role of EMT promoting Smad complexes. Cell Cycle 9: 2363-2374, 2010.

10. Li HX, Han M, Bernier M, Zheng B, Sun SG, Su M, Zhang R, $\mathrm{Fu}$ JR and Wen JK: Krüppel-like factor 4 promotes differentiation by transforming growth factor-beta receptor-mediated Smad and p38 MAPK signaling in vascular smooth muscle cells. J Biol Chem 285: 17846-17856, 2010.

11. Overdier DG, Porcella A and Costa RH: The DNA-binding specificity of the hepatocyte nuclear factor $3 /$ forkhead domain is influenced by amino-acid residues adjacent to the recognition helix. Mol Cell Biol 14: 2755-2766, 1994.

12. Hoggatt AM, Kriegel AM, Smith AF and Herring BP: Hepatocyte nuclear factor-3 homologue 1 (HFH-1) represses transcription of smooth muscle-specific genes. J Biol Chem 275: 31162-31170, 2000 .
13. Feuerborn A, Srivastava PK, Küffer S, Grandy WA Sijmonsma TP, Gretz N, Brors B and Gröne HJ: The Forkhead factor FoxQ1 influences epithelial differentiation. J Cell Physiol 226: 710-719, 2011

14. Qiao Y, Jiang X, Lee ST, Karuturi RK, Hooi SC and Yu Q: FOXQ1 regulates epithelial-mesenchymal transition in human cancers. Cancer Res 71: 3076-3086, 2011.

15. Zhang H, Meng F, Liu G, Zhang B, Zhu J, Wu F, Ethier SP, Miller $\mathrm{F}$ and $\mathrm{Wu} \mathrm{G}$ : Forkhead transcription factor foxq1 promotes epithelial-mesenchymal transition and breast cancer metastasis. Cancer Res 71: 1292-1301, 2011.

16. Sehrawat A, Kim SH, Vogt A and Singh SV: Suppression of FOXQ1 in benzyl isothiocyanate-mediated inhibition of epithelial-mesenchymal transition in human breast cancer cells. Carcinogenesis 34: 864-873, 2013.

17. Kaneda H, Arao T, Tanaka K, Tamura D, Aomatsu K, Kudo K, Sakai K, De Velasco MA, Matsumoto K, Fujita Y, et al: FOXQ1 is overexpressed in colorectal cancer and enhances tumorigenicity and tumor growth. Cancer Res 70: 2053-2063, 2010.

18. Feng J, Zhang X, Zhu H, Wang X, Ni S and Huang J: FoxQ1 overexpression influences poor prognosis in non-small cell lung cancer, associates with the phenomenon of EMT. PLoS One 7: e39937, 2012

19. Feng J, Xu L, Ni S, Gu J, Zhu H, Wang H, Zhang S, Zhang W and Huang J: Involvement of FoxQ1 in NSCLC through regulating EMT and increasing chemosensitivity. Oncotarget 5: 9689-9702, 2014.

20. Wang W, He S, Ji J, Huang J, Zhang S and Zhang Y: The prognostic significance of FOXQ1 oncogene overexpression in human hepatocellular carcinoma. Pathol Res Pract 209: 353-358, 2013.

21. Xia L, Huang W, Tian D, Zhang L, Qi X, Chen Z, Shang X, Nie Y and $\mathrm{Wu} \mathrm{K}$ : Forkhead box Q1 promotes hepatocellular carcinoma metastasis by transactivating ZEB2 and VersicanV1 expression. Hepatology 59: 958-973, 2014.

22. Pei Y, Wang P, Liu H, He F and Ming L: FOXQ1 promotes esophageal cancer proliferation and metastasis by negatively modulating CDH1. Biomed Pharmacother 74: 89-94, 2015.

23. Peng X, Luo Z, Kang Q, Deng D, Wang Q, Peng H, Wang S and Wei Z: FOXQ1 mediates the crosstalk between TGF- $\beta$ and Wnt signaling pathways in the progression of colorectal cancer. Cancer Biol Ther 16: 1099-1109, 2015.

24. Liang SH, Yan XZ, Wang BL, Jin HF, Yao LP, Li YN, Chen M, Nie YZ, Wang X, Guo XG, et al: Increased expression of FOXQ1 is a prognostic marker for patients with gastric cancer. Tumour Biol 34: 2605-2609, 2013.

25. Xiang XJ, Deng J, Liu YW, Wan LY, Feng M, Chen J and Xiong JP: MiR-1271 inhibits cell proliferation, invasion and EMT in gastric cancer by targeting FOXQ1. Cell Physiol Biochem 36: 1382-1394, 2015

26. Savagner P, Boyer B, Valles AM, Jouanneau J and Thiery JP: Modulations of the epithelial phenotype during embryogenesis and cancer progression. Cancer Treat Res 71: 229-249, 1994.

27. Kudo-Saito C, Shirako H, Takeuchi T and Kawakami Y: Cancer metastasis is accelerated through immunosuppression during Snail-induced EMT of cancer cells. Cancer Cell 15: 195-206, 2009. 